\title{
An acquired acute methemoglobinemia from dietary sources: Case reports and literature review
}

\author{
Angela Mauro, ${ }^{1}$ Iolanda Parente, ${ }^{1}$ Thailjlia Gagliardo, ${ }^{1}$ Anna Bonadies, ${ }^{1}$ Raffaele Mancusi, ${ }^{2}$ \\ Vincenzo Tipo, ${ }^{1}$ Eduardo Ponticiello ${ }^{1}$ \\ ${ }^{1}$ Pediatric Emergency Unit, Santobono-Pausilipon Children's Hospital, Naples; ${ }^{2}$ Department of Woman, \\ Child and of General and Specialized Surgery, Università degli Studi della Campania "Luigi Vanvitelli", \\ Naples, Italy
}

\begin{abstract}
Methemoglobinemia is an alteration of the oxidative state of hemoglobin. When methemoglobin values rise above $10 \%$, the symptoms and signs related to this condition appear, such as cyanosis, respiratory problems, fatigue and headache. Acquired methemoglobinemia is characterized by the sudden onset of cyanosis in a previous healthy child, which can be due to exposure to medications or chemical substances including nitrates, copper, sulfates, chlorites, chloramines and chlorates which can be present in food and water. We illustrate two cases of acquired methemoglobinemia related to nitrate ingestion from a vegetable source.
\end{abstract}

Correspondence: Angela Mauro, Pediatric Emergency Unit, SantobonoPausilipon Children's Hospital, Via Mario Fiore, Naples, Italy.

E-mail: angela.mauro84@gmail.com

Keys words: Methemoglobin; cyanosis; multiple-wavelength co-oximetry.

Contributions: AM and IP drafted the initial manuscript, and reviewed and revised the manuscript; TG, AB, RM, VT and EP acquired the data and critically revised the manuscript; All authors approved the final manuscript as submitted and are accountable for all aspects of the manuscript as submitted. Agreement to be accountable for all aspects of the work in ensuring that questions related to the accuracy or integrity of any part of the work are appropriately investigated and resolved.

Conflict of interest: No one. This work was not supported by any grant.

Availability of data and materials: All data generated or analyzed during this study are included in this published article.

Ethical approval and consent to participate: The patients' parents provided written consent for all of the investigations described in these reports, and for the reports themselves.

Received for publication: 7 May 2020.

Accepted for publication: 11 July 2020.

This work is licensed under a Creative Commons Attribution 4.0 License (by-nc 4.0).

${ }^{\circ}$ Copyright: the Author(s), 2021

Licensee PAGEPress, Italy

Emergency Care Journal 2021; 17:9089

doi:10.4081/ecj.2021.9089

\section{Introduction}

Methemoglobinemia is a rare blood cells disorder due to the hemoglobin iron oxidation from a ferrous to a ferric state. Methemoglobin (MET) cannot carry and release oxygen, with cyanosis and tissue hypoxemia as the consequence. Normal MET concentration in the blood is less than $2 \%{ }^{1}$

Methemoglobinemia can be congenital or acquired.

Congenital methemoglobinemia is characterized by early onset of cyanosis without any cardiac and pulmonary disease. Acquired methemoglobinemia is characterized by sudden cyanosis onset in a previous healthy child and can be due to exposure to medications, food and water. Acute diarrhea may also cause oxidation of hemoglobin to methemoglobin (Table 1). ${ }^{2}$

Although Acquired methemoglobinemia has been well described before, this represents a challenging condition for any clinicians. Indeed, in order to avoid long-term consequences and reduce the risk of repeated episodes, it is very important to identify this disease and carry out an accurate history to identify its etiology. ${ }^{3}$

We present two cases of acquired methemoglobinemia related to nitrate ingestion from a vegetable source, in order to remind practitioners that the acute onset of cyanosis can depend on other factors than cardiovascular and/or respiratory disease.

\section{Case Reports}

\section{Case \#1}

A 10-years-old boy was admitted to our emergency department for perioral cyanosis and bluish nail bed discoloration, associated with abdominal pain and tenderness. His mother reported an episode of lipothymia 30 minutes before. There was no history of significant family medical conditions or previous illnesses.

On admission, the patient was in good general conditions: blood pressure was $90 / 70 \mathrm{mmHg}$, heart rate $100 / \mathrm{min}$, respiratory rate $35 / \mathrm{min}$, oxygen saturation in room air $92 \%$, capillary refilling time was 2 " and body temperature $36^{\circ} \mathrm{C}$.

Physical examination showed a perioral cyanosis and bluish discoloration of the nail bed. Cardio-thoracic and abdominal examinations were unremarkable Electrocardiogram and chest X-ray showed no abnormalities, as well as there were no pathologic findings at echocardiography.

Blood tests revealed white blood cells $15,720 / \mathrm{mL}$ with normal differential count, platelet (PLT) 316,000/mL, hemoglobin ( $\mathrm{Hb}$ ) $13.9 \mathrm{~g} / \mathrm{dL}$, Hematocrit (Ht) $38.9 \%$, erythrocyte sedimentation rate (ESR) $15 \mathrm{~mm} / 1 \mathrm{~h}$ (normal value $20 \mathrm{~mm} / 1 \mathrm{~h}$ ), C-reactive protein (CRP) $1.3 \mathrm{mg} / \mathrm{L}$; The value of MET using multiple-wavelength 
CO-oximetry was $15 \%$, confirmed by the venous blood gas $(\mathrm{MET}=15 \%)$. During a further assessment of recent drug and diet history, the mother reported a significant assumptions of spinach during the last meal. The diagnosis of diet-induced methemoglobinemia was made and oxygen administration via nasal cannula was sufficient to treat this condition. After 2 hours of treatment, hemogasanalysis revealed significantly reduced MET levels $(1.5 \%)$. The patient was discharged on the following-day without prescription of any home-medication. He returned for a check-up a month later and was in good health.

\section{Case \#2}

An 8-months-old child was admitted to our emergency department with cyanosis and vomiting, suddenly onset about 40 minutes after his last meal. The child had no significant family medical conditions or past illnesses. His parents revealed that their son was not on any medication.

On admission, the patient was very irritable, with blood pressure at $85 / 60 \mathrm{mmHg}$, heart rate $150 / \mathrm{min}$, respiratory rate $45 / \mathrm{min}$, and oxygen saturation in room air $90 \%$, while capillary refilling time was 2 " and body temperature $36.3^{\circ} \mathrm{C}$. Physical examination showed perioral and finger area cyanosis. Cardiothoracic and abdominal examinations were unremarkable.

Most common causes of central and peripheral cyanosis were ruled out by echocardiography and electrocardiogram. Chest-X ray was normal. Blood samples were noticed to have a chocolate color (Figure 1), rising the suspect of acquired methemoglobinemia. On further interrogation, the mother revealed that the child has assumed a vegetable soup, mainly composed of tomatoes, spinach and potatoes which had been prepared two days before and kept in a refrigerator.

As per our hypothesis, multiple-wavelength CO-oximetry showed high MET levels (21\%), confirmed by the venous blood gas $(22 \%)$. All other blood tests were negative. The diagnosis of methemoglobinemia was made and the patient was treated with intravenous injection of methylene blue $(1 \mathrm{mg} / \mathrm{kg})$. After 1 hour, hemogasanalysis showed reduced MET $(0.8 \%)$, while oxygen saturation in room air was a steady $99 \%$. The patient was discharged two days later without any medications. After one month, he returned for the follow-up and was found to be in good health.

\section{Discussion}

Acquired methemoglobinemia is more frequently encountered than the congenital form ${ }^{4}$ and is frequently due to the exposure to hemoglobin oxidants, which overwhelm the cytochrome-b5 reductase's ability to reduce ferric hemoglobin. As a consequence, hemoglobin iron is transformed into ferric ion $\left(\mathrm{Fe}^{3+}\right)$ that is unable to transport oxygen. ${ }^{5}$ Common agents causing acquired methemoglobinemia include direct oxidizing agents (e.g. benzocaine and prilocaine), metabolic activation (e.g. aniline and dapsone) or indirect oxidation (e.g. nitrates). ${ }^{6}$

Ash-Bernal et al. described eleven pediatric patients among a retrospective series of 138 cases with acquired methemoglobinemia registered in two different hospitals. They showed that the most common causes of methemoglobinemia in their cohort were exposure to drugs and gastrointestinal infections with dehydration. Almost all (94\%) patients with methemoglobinemia were anemic. ${ }^{7}$

Drugs are widely described as a cause of methemoglobinemia, with the most common including dapsone and topical anesthetic agents.

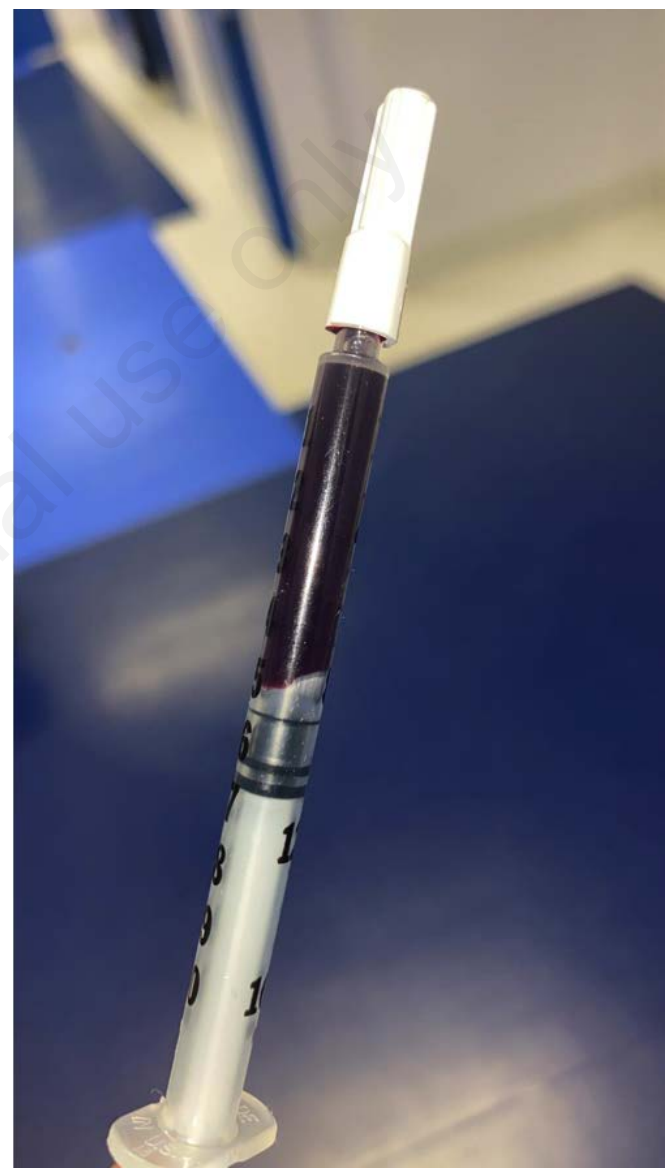

Figure 1. Chocolate color blood.

Table 1. Hereditary and acquired methemoglobinemia.

\begin{tabular}{ll} 
Hereditary methemoglobinemia & Acquired methemoglobinemia \\
Cytochrome b5-reductase deficiency & Local anesthetics: benzocaine, lidocaine \\
Hemoglobin M & Antimicrobials: chloroquin, dapsone, primaquin, sulfonamides \\
\hline & Analgesics: phenacetin, phenazopyridine \\
& Nitrates: contaminated well water or from diet. Fires and smoke inhalation. \\
& Other: aminophenol, aniline, broomates, chloratimetoclopramide, nitrobenezene, \\
chlorine benzene, nitroglycerin, nitrous oxides & Diarrhea \\
Acidosis
\end{tabular}


Dapsone is a drug used both as prophylactic therapy against Pneumocystis Carinii in severely immunocompromised patients and as therapy for leprosy and dermatitis herpetiformis. Dapsoneinduced methemoglobinemia may be dose-related or an idiosyncratic reaction in susceptible individuals. Chegondi et al. describe a case of a 4-years-old girl with end stage renal failure who developed a methaemoglobinaemia during therapy with Dapsone given as prophylactic therapy against Pneumocystis Carinii. ${ }^{8}$ After having been absorbed in the gut, this drug is metabolized into several metabolites, including dapsone hydroxylamine, an oxidant agent that can be responsible for methemoglobinemia and hemolytic anemia. In patients with renal failure, the impaired excretion of dapsone metabolites makes them more susceptible to methemoglobinemia.

A recent review from Guay et al. summarized 242 individual episodes of methemoglobinemia due to prilocaine or benzocaine use in 233 patients ( 9 repeated episodes) published between 1949 and 2007. These patients included 80 children (44 infants 6months-old or less). Forty patients had an intoxication related to Benzocaine and 37 to Prilocaine. ${ }^{9}$

In 2016, Cho et al. described a case of a 3.5-years-old child with seizures and methemoglobinemia after topical application of EMLA, an eutectic mixture of lidocaine and prilocaine widely used topically to provide local anesthesia for a variety of painful superficial procedures. ${ }^{10}$

Ingestion of nitrates is a rare cause of acquired methemoglobinemia in children. Usually, it is due to the ingestion of contaminated water or vegetables such as beets, spinach, peas and carrots. Variations in the chemical composition of plants can be linked both to season, soil composition, farming, and type and quantity of fertilizers used, or to inadequate transport or packaging conditions. $^{11}$

Sanchez-Echaniz et al. presented the clinical and epidemiological data regarding 7 infants with acquired methemoglobinemia related to consumption of vegetables. They mean age of patients was 8.14 months and none of the infants had diarrhea or was undernourished. In all cases, there was an history of assumption of homemade purée of mixed vegetables prepared in advance and kept in the refrigerator for 12 to 27 hours. Silver beets were a common ingredient and the methemoglobin levels ranged between $10 \%$ and $58 \%$. Three infants had nitrituria, which suggested the diagnosis of methemoglobinemia. ${ }^{12}$

Children at higher risk of intoxication are those below the age of 6 months, due to the reduced gastric acid production, a gut microbiota rich in nitrate-reducing bacteria, an immature methemoglobin reductase system, and the presence of certain amount of fetal hemoglobin that is more easily oxidized. ${ }^{13}$

Whereas in the second case described, both the age and the food history of the child quickly suggested the diagnosis, the first case that we described concerned a 10-years-old boy, which reminds us that, although unusual, this condition can occur at any age, especially in situations in which there is less availability of fresh food and therefore people consume more processed food.

It is actually well known that sodium nitrate and sodium nitrite are used for their antimicrobial effects to preserve and cure meat, fish, and certain cheeses. Few cases of methemoglobinemia due to accidental ingestion of high concentration of preservatives are described in literature. Kennedy et al. described a family outbreak of methemoglobinemia following ingestion of sausages made using 'saltpetre' a compound based on potassium and sodium used in cooking. ${ }^{14}$

It has to be taken in count that also the nasal inhalation of volatile nitrites can cause methemoglobinemia. ${ }^{15}$
The volatile nitrites are sweet-smelling liquids normally administered by nasal inhalation and commonly present in some illicit recreational drugs known as poppers. The nitrite present in poppers is amyl nitrite, an agent capable to dilate blood vessels inducing tachycardia and an euphoric feeling. In a study from 2000 and 2001 by National Household Surveys on Drug Abuse, 1.5\% of interviewed adolescents aged 12 to 17 years reported any lifetime use of nitrite inhalants. ${ }^{16}$

So, the intentional assumption of drugs should be considered when methemoglobinemia is suspected.

Physiologically, levels of methemoglobin are equal to $2 \%$ in term babies and less than $2 \%$ in adults. Cyanosis appears when methemoglobinemia levels are between $10 \%$ and $20 \%$. Values between $20 \%$ and $50 \%$ are associated with respiratory problems, dizziness, headache, and fatigue. Values above $60 \%$ can be lethal. ${ }^{17}$ On admission, our patients had methemoglobin levels of $15 \%$ and $22 \%$ respectively, presenting with mild symptoms.

If methemoglobin serum levels cannot be measured, the presence of an oxygen "saturation gap" between pulse oximetry and arterial blood gas analysis measurements is suggestive of methemoglobinemia. ${ }^{7}$ Indeed, pulse oximetry exclusively detects oxyhemoglobin and deoxyhemoglobin, while arterial blood gas analysis detects also methemoglobin, thus providing higher saturation readings. When the observed gap is superior to 5 percentage saturation points, the presence of forms others than oxy- and deoxyhemoglobins should be suspected (i.e. methemoglobin, carboxyemoglobin). ${ }^{18}$

Moreover, in presence of low saturation registered at standard pulse oximetry, an arterial blood gas sample should be always performed.

However, in 2005, Masimo Corp. developed the new 'Rainbow Technology' pulse oximeters permitting the non-invasive measurement of carboxyhemoglobin, methemoglobin, and total hemoglobin as this device uses eight wavelengths of light corresponding to specific characteristic of absorbance values of derivatives of hemoglobin. The additional wavelengths improve the accuracy of the spectrum, minimizing or eliminating the interfering substances and better detect the presence of the dishemoglobin. So, when available, multiple-wavelength co-oximetry is the best choice to diagnose methemoglobinemia. ${ }^{17}$

Forms with methemoglobin $<20 \%$ can be treated by oxygen administration and elimination of the source of contamination from the diet. In symptomatic patients with methemoglobin levels higher than $20 \%$, or in presence of MET levels higher than $30 \%$, treatment with methylene blue administration is suggested. ${ }^{19}$

Methylene blue, slowly injected intravenously at the dose of 1$2 \mathrm{mg} / \mathrm{Kg}$, combines with nicotinamide adenine dinucleotidephosphatereduced (NADPH), in the presence of NADPH-methemoglobinreductase, to produce leukomethylene blue which then reduces the heme group acting as cofactor to accelerate the conversion of methemoglobin to hemoglobin in erythrocytes. Methylene blue can reduce the half life of methemoglobin from hours to minutes. Because glucose 6 phosphate dehydrogenase (G6PD) is required to regenerate NADPH, Methylene blue therapy is ineffective in individuals with G6PD deficiency. Moreover, in these patients Methylene Blue can cause severe hemolysis itself an oxidantagent. ${ }^{20}$

\section{Conclusions}

In conclusion, acquired methemoglobinemia should be consid- 
ered in case of acute cyanosis in children with no respiratory symptoms or cardiovascular disease. A good history and high level of suspicion are required to make the diagnosis and the use of multiple-wavelength co-oximetry can be a non-invasive instruments for the diagnosis of methemoglobinemia.

\section{References}

1. Baraka AS, Ayoub CM, Yazbeck-Karam V, et al. Prophylactic methylene blue in a patient with congenital methemoglobinemia. Can J Anaesth 2005;52:258-61.

2. Meier J, Pape A, Lauscher P, et al. Hyperoxia in lethal methemoglobinemia: Effects on oxygen transport, tissue oxygenation, and survival in pigs. Crit Care Med 2005;33:1582-8.

3. Filer LJ, Lower CU, \& Barness LA: Infant methemoglobinemia. The role of dietary nitrate. Pediatrics 1970;46:475-8.

4. Curry S. Methemoglobinemia. Ann Emerg Med 1982;11:21421.

5. Ludlow JT, Wilkerson RG, Nappe TM. Methemoglobinemia. StatPearls [Internet]. Treasure Island (FL): StatPearls Publishing; 2020 Jan. 2020 Sep 4.

6. Bradberry SM. Occupational methaemoglobinaemia. Mechanisms of production, features, diagnosis and management including the use of methylene blue. Toxicol Rev 2003;22:13-27.

7. Ash-Bernal R, Wise R, Wright SM. Acquired methemoglobinemia: a retrospective series of 138 cases at 2 teaching hospitals. Med (Baltimore) 2004;83:265-73.

8. Chegondi M, Ten I, Totapally B. Dapsone-induced Methemoglobinemia in a Child with End-stage Renal Disease: A Brief Review. Cureus 2018; 10:e2513.

9. Guay J. Methemoglobinemia Related to Local Anesthetics: A Summary of 242 Episodes. Anesth Analg 2009;108:837-45.
10. Cho YS, Chung BY, Park CW, Kim HO. Seizures and Methemoglobinemia After Topical Application of Eutectic Mixture of Lidocaine and Prilocaine on a 3.5-Year-Old Child with Molluscum Contagiosum and Atopic Dermatitis. Pediatr Dermatol 2016;33:e284-5.

11. Martínez de Zabarte Fernández JM, García Íñiguez JP, Domínguez Cajal M. Metahemoglobinemia in infants over one year. Med Clin (Barc) 2018;151:278-80.

12. Sanchez-Echaniz J, Benito-Fernández J, Mintegui-Raso S. Methemoglobinemia and consumption of vegetables in infants. Pediatrics 2001;107:1024-8.

13. Cannata G, Abate L, Scarabello C, et al. The Dose Makes the Poison: A Case Report of Acquired Methemoglobinemia. Int J Environ Res Public Health 2020;17.

14. Kennedy N, Smith CP, McWhinney P. Faulty sausage production causing methaemoglobinaemia. Arch Dis Child 1997;76:367-8.

15. Sutton M, Jeffrey B. Acquired acute methaemoglobinaemia from amyl nitrite inhalation. J Emerg Nurs 1992;18:8-9.

16. Wu L-T, Schlenger WE, Ringwalt CL. Use of nitrite inhalants ("poppers") among American youth. J Adolesc Health 2005;37:52-60.

17. Shamir MY, Avramovich A, Smaka T. The current status of continuous noninvasive measurement of total, carboxy, and methemoglobin concentration. Anesth Analg 2012;114:972-8

18. Skold A, Cosco DL, Klein R. Methemoglobinemia: Pathogenesis, diagnosis, and management. South Med J 2011;104:757-61.

19. Cortazzo JA, Lichtman AD. Methemoglobinemia: A Review and recommendations for management. J Cardiothorac Vasc Anesth 2014;28:1043-7.

20. Erstad BL. Dapsone-induced methemoglobinemia and hemolytic anemia. Clin Pharm 1992;11:800-5. 